\title{
49.
}

\section{SUR QUELQUES FORMULES DU CALCUL INTÉGRAL.}

[From the Journal de Mathématiques Pures et Appliquées (Liouville), tom. XII. (1847), pp. $231-240$.]

SorT $x+y \sqrt{-1}$ ou $x+i y$ une quantité imaginaire quelconque; faisons

$$
\rho=\sqrt{x^{2}+y^{2}}, \quad \theta=\arctan \frac{y}{x}
$$

$\rho$ étant une quantité positive, et $\theta$ un arc compris entre les limites $\frac{1}{2} \pi,-\frac{1}{2} \pi$. Cela posé, écrivons

$$
\left.\begin{array}{ll}
(x+i y)^{m}=\rho^{m} e^{i m \theta} & (x \text { positif }) \\
(x+i y)^{m}=\rho^{m} e^{i m(\theta \pm \pi)} & (x \text { négatif })
\end{array}\right\}
$$

(dans la seconde de ces formules, il faut prendre le sigrie supérieur ou inférieur, selon que $y$ est positif ou négatif.) Au cas de $x$ positif, la valeur du second membre sera ce que M. Cauchy a appelé valeur principale de $(x+i y)^{m}$. Au cas de $x$ négatif, on peut aussi, à ce qu'il me semble, nommer cette valeur valeur principale. Cela paraît contraire à la théorie de M. Cauchy (Exercices de Mathématiques, t. I. [1826] p. 2); mais la démonstration que l'on y trouve de l'impossibilité d'une valeur principale pour $x$ négatif ne s'applique qu'au cas où l'on suppose que le signe \pm est toujours le même sans avoir égard au signe de $y$. Seulement, selon nos définitions, il importe de remarquer qu'il n'y a pas de valeur principale pour $x$ négatif, au cas particulier où $y=0$; ou plutôt dans ce cas, et dans ce cas seulement, la valeur principale devient indéterminée.

Soit, en partiçulier, $x=0$; les deux formules conduisent au même résultat, savoir

$$
(i y)^{m}=( \pm y)^{m} e^{ \pm \frac{1}{2} m \pi i}
$$


le signe comme auparavant. Car en considérant $x$ comme infiniment petit positif, on obtient

$$
\theta= \pm \frac{1}{2} \pi
$$

et, en considérant $x$ comme infiniment petit négatif,

$$
\theta=\mp \frac{1}{2} \pi \text {, }
$$

et de là

$$
\theta \pm \pi= \pm \frac{1}{2} \pi
$$

Ainsi cette formule est toujours vraie, sans qu'il soit nécessaire de considérer iy comme limite de $x+i y, x$ positif ou $x$ négatif.

Remarquons encore que cette fonction $(i y)^{m}$ reste continue quand y passe par zéro, ce qui a lieu aussi pour $(x+i y)^{m}, x$ positif, mais non pas pour $(x+i y)^{m}, x$ négatif.

Les mêmes remarques s'appliquent aux valeurs principales des logarithmes, lesquelles doivent se définir d'une manière analogue par les équations

$$
\left.\begin{array}{ll}
\log (x+i y)=\log \rho+i \theta & (x \text { positif }), \\
\log (x+i y)=\log \rho+i(\theta \pm \pi) & (x \text { négatif }),
\end{array}\right\}
$$

le signe ambigu, comme auparavant. équations

On démontre sans difficulté que ces valeurs principales satisfont en tout cas aux

$$
\left.\begin{array}{rl}
(x+i y)^{m}\left(x^{\prime}+i y^{\prime}\right)^{m} & =\left[(x+i y)\left(x^{\prime}+i y^{\prime}\right)\right]^{m}, \\
\log (x+i y) \log \left(x^{\prime}+i y^{\prime}\right) & =\log \left[(x+i y)\left(x^{\prime}+i y^{\prime}\right)\right]
\end{array}\right\}
$$

seulement ces équations deviennent indéterminées au cas où, l'une des quantités $x, x^{\prime}, x x^{\prime}-y y^{\prime}$ étant négative, la quantité correspondante $y, y^{\prime}, x y^{\prime}+x^{\prime} y$ s'évanouit.

Au moyen de cette définition de la valeur principale d'un logarithme, on obtient

$$
\int_{\beta}^{a} \frac{d x}{A+B x}=\log \left(\frac{A+B \alpha}{A+B \beta}\right)
$$

où $\alpha, \beta$ sont réels, et $A, B$ sont assujettis à la seule condition qu'au cas où $\alpha, \beta$ seraient de signes contraires, la partie imaginaire de $\frac{A}{B}$ ne s'évanouisse pas. En effet, dans ce cas, l'intégrale et la valeur principale du logarithme deviennent toutes les deux indéterminées. Sans doute il y a une valeur que l'on peut appeler principale de l'intégrale, mais cette valeur n'est égale à aucun des logarithmes de $\frac{A+B \alpha}{A+B \beta}$, et les notions des valeurs principales d'une intégrale et d'un logarithme n'ont pas de rapport ensemble. Ce résultat s'accorde avec celui que j'ai trouvé dans mon Mémoire "Sur les fonctions doublement périodiques," [25]. 
Je passe à quelques autres applications de ces principes, qui ont rapport à la théorie des fonctions $\Gamma$. Soit d'abord $r$ un nombre positif plus petit que l'unité, et écrivons

$$
U=\int_{-\infty}^{\infty}(i x)^{r-1} e^{i x} d x
$$

on obtient

$$
U=\int_{0}^{\infty}(i x)^{r-1} e^{i x} d x+\int_{0}^{\infty}(-i x)^{r-1} e^{-i x} d x,
$$

ou enfin, puisque $x$ est positif dans ces deux intégrales,

$$
U=e^{\frac{1}{2}(r-1) \pi i} \int_{0}^{\infty} x^{r-1} e^{i x} d x+e^{-\frac{1}{2}(r-1) \pi i} \int_{0}^{\infty} x^{r-1} e^{-i x} d x ;
$$

au moyen de la formule connue,

$$
\int_{0}^{\infty} x^{r-1} e^{ \pm i x} d x=e^{ \pm \frac{1}{2} r \pi i} \Gamma r
$$

on en conclut

$$
U=2 \cos \left(r-\frac{1}{2}\right) \pi . \Gamma r=2 \sin r \pi . \Gamma r,
$$

savoir

$$
\sin r \pi . \Gamma r=\frac{1}{2} \int_{-\infty}^{\infty}(i x)^{r-1} e^{i x} d x
$$

On obtiendrait de même

$$
0=\frac{1}{2} \int_{-\infty}^{\infty}(-i x)^{r-1} e^{i x} d x .
$$

$\mathrm{Au}$ premier coup d'œil, ces équations pourraient paraître en contradiction l'une avec l'autre: mais cela n'est pas ainsi, parce qu'il n'est pas vrai que $(-i x)^{r-1}$ soit égal à $(-1)^{r-1}(i x)^{r-1},(-1)^{r-1}$ étant facteur invariable; mais au contraire $(-i x)^{r-1}=e^{ \pm(r-1) \pi i}(i x)^{r-1}$, selon que $x$ est positif ou négatif.

Dans la première de ces équations, on peut remplacer $i x$ par $c+i x$, et dans la seconde, $-i x$ par $c-i x$, $c$ étant positif; mais cela n'est pas permis pour $c$ négatif, à cause de la discontinuité de valeur de $(c+i x)^{r-1}$ ou $(c-i x)^{r-1}$ dans ce cas, quand $x$ passe par zéro. En multipliant la première équation par $e^{-c}$, et différentiant un nombre quelconque de fois, en admettant, pour les valeurs négatives de $r$, l'équation

$$
\Gamma(r+1)=r \Gamma r,
$$

la formule ne change pas de forme, et l'on obtient

$$
\sin r \pi \Gamma r \cdot e^{-c}=\frac{1}{2} \int_{-\infty}^{\infty}(c+i x)^{r-1} e^{i x} d x
$$

et de même, au moyen de la seconde équation,

$$
0=\frac{1}{2} \int_{-\infty}^{\infty}(c-i x)^{r-1} e^{i x} d x
$$


dans lesquelles formules $c$ est positif, et $r$ est un nombre quelconque entre $1,-\infty$, sans exclure la limite supérieure; seulement, pour $r=0$, le facteur $\sin r \pi \Gamma r$ se réduit à $\pi$. Au cas où $r$ est plus grand que zéro, on peut, si l'on veut, écrire aussi $c=0$. Dans tous les cas, on peut remplacer sin $r \pi \Gamma r$ par $\frac{\pi}{\Gamma(1-r)}$. Il importe de remarquer que ces mêmes intégrales sont absolument inexprimables au cas où $c$ est négatif: en effet, en écrivant $-c$ au lieu de $c$ ( $c$ positif), on obtiendrait

$$
\int_{-\infty}^{\infty}(-c+i x)^{r-1} e^{i x} d x=e^{(r-1) \pi i} \int_{0}^{\infty}(c-i x)^{r-1} e^{i x} d x+e^{-(r-1) \pi i} \int_{-\infty}^{0}(c-i x)^{r-1} e^{i x} d x .
$$

Mais, par la seconde des équations dont il s'agit,

$$
0=\int_{0}^{\infty}(c-i x)^{r-1} e^{i x} d x+\int_{-\infty}^{0}(c-i x)^{r-1} e^{i x} d x
$$

donc

$$
\int_{-\infty}^{\infty}(-c+i x)^{r-1} e^{i x} d x=-2 i \sin r \pi \int_{0}^{\infty}(c-i x)^{r-1} e^{i x} d x .
$$

Or l'intégrale au second membre ne peut pas s'exprimer par les transcendantes connues, à moins que $r$ ne soit entier. Écrivons encore, $c$ étant toujours positif,

$$
\left.\begin{array}{l}
I=\int_{0}^{\infty}(c+i x)^{r-1} e^{i x} d x \\
I_{1}=\int_{0}^{\infty}(c-i x)^{r-1} e^{i x} d x \\
I_{2}=\int_{0}^{\infty}(c+i x)^{r-1} e^{-i x} d x \\
I_{3}=\int_{0}^{\infty}(c-i x)^{r-i} e^{-i x} d x
\end{array}\right\}
$$

Toutes les fonctions

$$
\int( \pm c \pm i x)^{r-1} e^{ \pm i x} d x
$$

entre les limites $0, \infty$, ou $-\infty, 0$, ou $-\infty, \infty$, s'expriment facilement au moyen de $I, I_{1}, I_{2}, I_{3}$. Mais ces quatre fonctions ne sont pas connues; seulement, au moyen des équations qui viennent d'être trouvées, on obtient

$$
\left.\begin{array}{rl}
2 \sin r \pi \Gamma r . e^{-c} & =I+I_{3}, \\
0 & =I_{1}+I_{2} .
\end{array}\right\}
$$

On déduit encore de ces mêmes formules:

$$
\left.\begin{array}{c}
\sin r \pi \Gamma r \cdot e^{-c} \\
=\int_{-\infty}^{\infty}(c+i x)^{r-1} \cos x d x=\quad i \int_{-\infty}^{\infty}(c+i x)^{r-1} \sin x d x, \\
=\int_{-\infty}^{\infty}(c-i x)^{r-1} \cos x d x=-i \int_{-\infty}^{\infty}(c-i x)^{r-1} \sin x d x .
\end{array}\right\}
$$


En supposant que $r-1$ est entier négatif, l'intégrale

$$
\int_{0}^{\infty}\left(c^{2}+x^{2}\right)^{r-1} \cos x d x
$$

se décompose facilement dans une suite d'intégrales de la forme

$$
\int_{-\infty}^{\infty}(c \pm i x)^{p-1} \cos x d x
$$

et, en prenant la somme de celles-ci, on obtient la formule

$$
\int_{0}^{\infty}\left(c^{2}+x^{2}\right)^{r-1} \cos x d x=\frac{\pi e^{-c}(2 c)^{2 r-1}}{\Gamma^{2}(1-r)} \int_{0}^{\infty} \theta^{-r}(\theta+2 c)^{-r} e^{-\theta} d \theta \ldots \ldots \ldots
$$

laquelle est due à M. Catalan. Cependant, ni cette démonstration ni celie de M. Catalan ne s'appliquent au cas où $r$ n'est pas entier; la formule subsiste encore dans ce cas, comme M. Serret l'a démontré rigoureusement. En essayant de la vérifier, je suis tombé sur cette autre formule:

$$
\left.\begin{array}{c}
\int_{0}^{\infty}\left(c^{2}+x^{2}\right)^{r-1} \cos x d x \\
=\frac{\sqrt{\pi} e^{-c}(2 c)^{2 r-1}}{2 \Gamma(1-r)} \int_{0}^{\infty} \frac{(\sqrt{\theta+2 c}+\sqrt{\theta})^{1-2 r}+(\sqrt{\theta+2 c}-\sqrt{\theta})^{1-2 r}}{\sqrt{ } \bar{\theta} \sqrt{\theta+2 c}} e^{-\theta} d \theta
\end{array}\right\}
$$

ce qui suppose, comme auparavant, que $\overline{r-1}$ soit négatif; en comparant les deux valeurs, on est conduit au résultat singulier (en écrivant $\alpha$ au lieu de $2 c$, et $\frac{1}{2}(1-p)$ pour $r$ ):

$$
\left.\begin{array}{c}
\int_{0}^{\infty} \frac{(\sqrt{\theta+\alpha}+\sqrt{\theta})^{p}+(\sqrt{\theta+\alpha}-\sqrt{\theta})^{p}}{\sqrt{\theta} \sqrt{\theta+\alpha}} e^{-\theta} d \theta \\
=\frac{2 \sqrt{\pi}}{\Gamma_{\frac{1}{2}}(p+1)} \int_{0}^{\infty} \theta^{\frac{1}{2}(p-1)}(\theta+\alpha)^{\frac{1}{2}(p-1)} e^{-\theta} d \theta
\end{array}\right\}
$$

lequel peut se démontrer sans difficulté, quand $p$ est entier positif impair, en développant les deux membres suivant les puissances de $\alpha$; cela se fait au moyen de

$$
\frac{(\sqrt{1+x}+1)^{p}+(\sqrt{1+x}-1)^{p}}{\sqrt{1+x}}=\boldsymbol{S}_{r}\left[x^{r} \frac{2^{p-2 r} \Gamma(p-r)}{\Gamma(p-2 r) \Gamma(r+1)}\right] .
$$

où $r$ s'étend depuis 0 jusqu'à $\frac{1}{2}(p-1)$. J'ai déduit de cette formule (14) des formules assez remarquables qui se rapportent aux attractions, lesquelles paraîtront dans un numéro prochain du Cambridge and Dublin Mathematical Journal, [41]. On peut encore démontrer cette formule singulière:

$$
\Gamma(r+a-1)=\frac{1}{2 \sin r \pi} \int_{-\infty}^{\infty}(i x)^{r-1}(-i x)^{a-1} e^{i x} d x
$$

C. 
en effet, pour la vérifier, il suffit de réduire l'intégrale, d'abord à

$$
\int_{0}^{\infty}(i x)^{r-1}(-i x)^{a-1} e^{i x} d x+\int_{0}^{\infty}(-i x)^{r-1}(i x)^{a-1} e^{-i x} d x
$$

puis à

$$
e^{\frac{1}{2}(r-a) \pi i} \int_{0}^{\infty} x^{r+a-2} e^{i x} d x+e^{\frac{1}{2}(a-r) \pi i} \int_{0}^{\infty} x^{r+a-2} e^{-i x} d x,
$$

dont les deux parties s'obtiennent au moyen d'une formule donnée ci-dessus. Si, au lieu de $(-i x)^{a-1}$, l'on avait $(i x)^{a-1}$, l'intégrale se réduirait à zéro; mais cela rentre dans une formule plus simple.

J'ajouterai encore ces deux formules-ci,

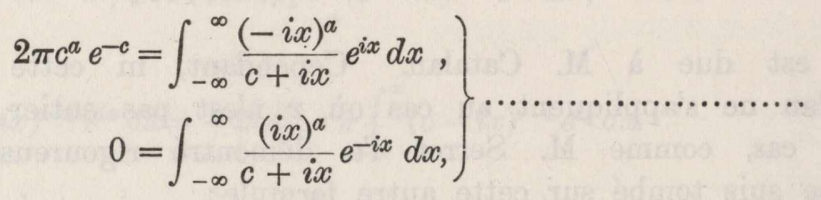

dont je supprime la démonstration. En écrivant dans la dernière $-x$ au lieu de $x$, puis ajoutant, on a

$$
\pi c^{a-1} e^{-c}=\int_{-\infty}^{\infty} \frac{(-i x)^{a}}{c^{2}+x^{2}} e^{i x} d x
$$

d'où l'on déduit tout de suite cette formule de M. Cauchy

$$
\frac{1}{2} \pi c^{a-1} e^{-c}=\int_{0}^{\infty} \frac{x^{a}}{c^{2}+x^{2}} \cos \left(\frac{1}{2} a \pi-x\right) d x
$$

La formule (7) peut être considérée comme une définition de la fonction $\Gamma r$ au cas de $r$ négatif; et, à ce point de vue, elle a, ce me semble, quelques avantages sur celle que M. Cauchy a donnée au moyen des intégrales extraordinaires. Je passe à la. définition, au moyen d'une intégrale définie, de la seconde intégrale eulérienne,

$$
\mathscr{F}(m, n)=\frac{\Gamma m \Gamma n}{\Gamma(m+n)}
$$

quand $m$ ou $n$, ou tous les deux, sont négatifs. Soit d'abord $m$ et $n$ tous les deux positifs, mais $n$ plus petit que l'unité; on obtient au moyen de la formule (7) et de la définition ordinaire des fonctions $\Gamma$,

$$
\Gamma m \Gamma n=\frac{1}{2 \sin n \pi} \int_{0}^{\infty} d x \int_{-\infty}^{\infty} d y x^{m-1}(i y)^{n-1} e^{-x+i y},
$$

et de là, en mettant

$$
y=\alpha x, \quad d y=x d \alpha
$$

et intégrant par rapport à $x$,

$$
\mathscr{F}(m, n)=\frac{1}{2 \sin n \pi} \int_{-\infty}^{\infty} \frac{(i \alpha)^{n-1} d \alpha}{(1-i \alpha)^{m+n}},
$$


ou en écrivant $k+\alpha i$ au lieu de $\alpha i, k$ étant positif,

$$
\mathfrak{F}(m, n)=\frac{1}{2 \sin n \pi} \int_{-\infty}^{\infty} \frac{(k+\alpha i)^{n-1} d \alpha}{(1-k-a i)^{m+n}}
$$

formule qui est vraie, même pour les valeurs négatives de $n$. En effet, si cette formule est vraie pour une valeur quelconque particulière de $n$, elle sera aussi vraie pour la valeur $n+p, p$ étant entier positif quelconque; ce qui se démontre au moyen des formules de réduction: donc il ne s'agit que de la démontrer au cas où $n$ est positif et plus petit que l'unité, et dans ce cas, puisque la fonction à intégrer est toujours finie, on peut écrire sans crainte $k+\alpha i$ au lieu de ai, ce qui la réduit à la formule qui vient d'être démontrée; donc cette formule (21) est la définition cherchée, au cas où $n$ est négatif, ou positif et plus petit que l'unité.

$\mathrm{Si}$, de plus, $m$ est négatif, ou positif et plus petit que l'unité, $l-m$ sera positif, et l'on déduira

$$
\mathfrak{F}(m, n)=\frac{\Gamma m \Gamma n}{\Gamma(m+n)}=\frac{\Gamma(1-m-n) \Gamma n}{\Gamma(1-m)} \frac{\sin (m+n) \pi}{\sin m \pi}
$$

c'est-à-dire

$$
\mathfrak{F}(m, n)=\frac{\sin (m+n) \pi}{\sin m \pi} \mathfrak{F}(1-m-n, n)
$$

ou enfin, par l'équation (21),

$$
\mathfrak{F}(m, n)=\frac{\sin (m+n) \pi}{2 \sin m \pi \sin n \pi} \int_{-\infty}^{\infty}(k+i \alpha)^{n-1}(1-k-i x)^{m-1} d \alpha,
$$

laquelle suppose seulement que $m+n$ soit négatif, ou positif et plus petit que l'unité. Elle présente une analogie assez frappante avec l'équation ordinaire

$$
\mathfrak{F}(m, n)=\int_{0}^{1} \alpha^{n-1}(1-\alpha)^{m-1} d \alpha
$$

qui correspond aux valeurs positives de $m, n$; de même que l'équation (21) est analogue à cette autre forme

$$
\mathfrak{F}(m, n)=\int_{0}^{\infty} \frac{a^{n-1} d \alpha}{(1+\alpha)^{m-n}}
$$

qui correspond aussi aux valeurs positives de $m$ et $n$.

On peut se proposer de vérifier l'équation ( $m$ et $n$ positifs et plus petits que l'unité)

$$
\Gamma m \Gamma n=\frac{1}{4 \sin m \pi \sin n \pi} \int_{-\infty}^{\infty} d x \int_{-\infty}^{\infty} d y(i x)^{m-1}(i y)^{n-1} e^{i(x+y)},
$$

en transformant le second membre au moyen de $x=a y$. Pour cela, on distinguera quatre cas, selon que $x$ et $y$ sont tous les deux positifs ou négatifs, ou l'un positif et l'autre négatif. En mettant dans les deux premiers

$$
x=\alpha y, \quad d x=y d \alpha,
$$


et en intégrant par rapport à $y$, on trouvera que les deux portions correspondantes de l'intégrale double se réuniront en

$$
-\Gamma(m+n) \cdot 2 \cos (m+n) \pi \int_{0}^{\infty} \frac{\alpha^{m-1} d \alpha}{(1+\alpha)^{m+n}},
$$

c'est-à-dire à

$$
-2 \cos (m+n) \pi \Gamma m \Gamma n .
$$

Pour les deux autres portions de l'intégrale double, en écrivant

$$
x=-\alpha y,
$$

on verra qu'il faut encore distinguer les deux cas $\alpha<1$ et $\alpha>1$. Les quatre intégrales ainsi obtenues se réuniront cependant dans les deux

$$
2 \cos n \pi \Gamma(m+n) \int_{0}^{1} \frac{\alpha^{m-1} d \alpha}{(1-\alpha)^{m+n}}, \quad 2 \cos m \pi \int_{1}^{\infty} \frac{\alpha^{m-1} d \alpha}{(\alpha-1)^{m+n}},
$$

savoir, après quelques réductions faciles, dans celles-ci,

$$
\frac{2 \cos n \pi \sin m \pi}{\sin (m+n) \pi} \Gamma m \Gamma n, \quad \frac{2 \cos m \pi \sin m \pi}{\sin (m+n)} \Gamma m \Gamma n,
$$

lesquelles se réuniront en

$$
\cos (m-n) \pi \Gamma m \Gamma n .
$$

On obtient donc enfin l'équation identique

$$
4 \sin m \pi \sin n \pi=2 \cos (m-n) \pi-2 \cos (m+n) \pi ;
$$

ce qui suffit pour la vérification dont il s'agit. 